blood compounds, including cholesterol, C-reactive protein and fibrinogen, have been linked with increased incidence of CAV, some patients have been treated with statins and heparin-mediated extracorporeal LDL/ fibrinogen precipitation (HELP)-apheresis following heart transplantation. Although the efficacy of HELP-apheresis treatment regarding reduction of these blood compounds is well known, its effect on myocardial blood flow and transplant outcome has not been investigated fully.

Jaeger et al. compared myocardial tissue blood flow before and after apheresis in 10 patients who had survived a heart transplant for an average of 5.6 years. Angiography showed that six patients had signs of CAV and one patient had a prominent calcified plaque in the left anterior descending artery; the other patients had normal angiographic findings.

Following a single HELP-apheresis treatment, there were $42-48 \%$ reductions in patients' cholesterol, fibrinogen, lipoprotein (a) and C-reactive protein levels. Positron emission tomography heart scans also revealed an increase in median hyperemic and coronary flow reserves.

This study provides supporting evidence that HELP-apheresis can improve myocardial blood flow in transplanted heart tissue. The authors hypothesize that, by reducing fibrinogen, plasma viscosity and erythrocyte aggregation, more oxygen can be delivered to the heart tissue, thereby improving microvascular blood flow and ischemic tolerance.

Claire Braybrook

Original article Jaeger et al. (2005) Changes in myocardial vasoreactivity after drastic reduction of plasma fibrinogen and cholesterol: a clinical study in long-term heart transplant survivors using positron emission tomography. J Heart Lung Transplant [doi: 10.1016/j.healun.2005.05.009]

\section{Intravascular MRI for the characterization of deep-vessel atherosclerotic plaques}

There is currently a need for imaging techniques that can successfully detect the presence, prognosis and progress of atherosclerotic plaques, especially in deeper arteries. One such method, intravascular MRI (IVMRI), has shown promise in ex vivo models.
Larose and colleagues first validated IVMRI against histopathology in atherosclerotic arteries ex vivo. Lipid, fibrous and calcified components within the plaque were successfully differentiated with good sensitivity and selectivity by combined T1-weighted, moderate T2weighted and proton-density-weighted imaging. Accurate assessment of plaque size was also achieved. The technique was then assessed by imaging the iliac arteries of 25 patients in vivo, and results were compared with intravascular ultrasound (IVUS). By use of IVMRI, inner and outer plaque boundaries were readily visualized in all plaques, including those with heavy calcification; the heterogeneous structure of the plaques was also detectable. By contrast, IVUS did not detect this heterogeneity and was unable to measure size in calcified plaques because of interference. Interobserver and intraobserver agreement was high for the determination of plaque composition using IVMRI, but was poor for IVUS.

With IVMRI, the composition and size of plaques within deep vessels such as the iliac artery can be reliably determined. The authors suggest that this information might prove useful in research into new therapies for plaque stabilization. Although further work is needed to improve the associated technology, IVMRI shows potential as an investigative and clinical tool in atherosclerosis.

Carol Lovegrove

Original article Larose E et al. (2005) Characterization of human atherosclerotic plaques by intravascular magnetic resonance imaging. Circulation 112: 2324-2331

\section{Bezafibrate decreases the incidence and delays the onset of type 2 diabetes}

Type 2 diabetes is a major global health problem that could be prevented by lifestyle changes and pharmacologic therapies that alter primary lipid metabolism. Bezafibrate is a fibrate derivative with triglyceride-lowering and HDL-cholesterol-raising properties that can reduce the incidence of diabetes in patients with impaired fasting glucose levels.

In a post hoc analysis of a multicenter prospective trial, Tenenbaum et al. evaluated the onset of type 2 diabetes in nondiabetic obese patients $\left(\mathrm{BMl} \geq 30.0 \mathrm{~kg} / \mathrm{m}^{2}\right)$ with normal fasting glucose 